\title{
Vers une gestion autonome de la médication en psychiatrie ? Approche anthropologique
}

\section{Towards an autonomous management of medication in psychiatry? An anthropological approach}

\author{
J. Palazzolo $^{\text {a,de, }, *}$, N. Midol ${ }^{\text {b }}$, J. Candau $^{\text {c }}$

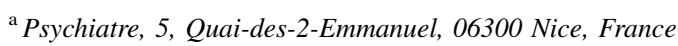 \\ ${ }^{\mathrm{b}}$ Maître de conférences \\ ${ }^{\mathrm{c}}$ Professeur d'anthropologie, LASMIC, UFR Lettres, Arts et Sciences Humaines, 98, Boulevard Edouard-Herriot, 06204 Nice cedex 03, France \\ ${ }^{\mathrm{d}}$ Professeur de socio-anthropologie de la santé, Université internationale Senghor, Alexandrie, Égypte \\ ${ }^{\mathrm{e}}$ Chercheur associé au LASMIC, France \\ Reçu le 28 décembre 2005 ; accepté le 10 février 2006 \\ Disponible sur Internet le 15 février 2007
}

\section{Résumé}

Du point de vue du patient, la gestion autonome de la médication passe par l'obtention d'une bonne relation avec son médecin ; cette dernière est directement liée à l'écoute et à l'intérêt accordés au malade, et pas seulement à ses symptômes. Si la motivation individuelle joue un rôle important au départ, elle va être elle-même fortement influencée par la relation médecin-malade. C'est l'un des rares facteurs pour lequel on observe une corrélation positive avec le respect de l'ordonnance, comme ont pu le constater pratiquement toutes les recherches en psychologie médicale. Mais, de telles données témoignent du fossé existant entre ce qui est attendu et ce qui est effectivement réalisé. Cette divergence intervient autant pour le patient que pour son thérapeute, et fait intervenir de nombreux autres facteurs appartenant à la culture, au contexte médicoreligieux, à l'anthropologie médicale, aux propriétés réelles ou supposées du Pharmakon... Notre travail se propose de faire la synthèse des données les plus actuelles concernant cette problématique, encore objet de nombreux débats et quasiment pas abordée au sein de la littérature francophone. Grâce à la synthèse de diverses données théoriques et aux témoignages de 135 personnes impliquées dans cette dynamique (patients, familles, médecins, infirmiers, membres de l'industrie pharmaceutique), nous essayerons de mieux comprendre ce qui se passe entre le soigné et ses soignants, d'un point de vue anthropologique.

(C) 2007 Elsevier Masson SAS. Tous droits réservés.

\begin{abstract}
From the patient's viewpoint, a good quality of compliance supposes a good relationship with his/her doctor; this relationship is directly linked to the heeding and the interest shown to the patient, and not to his/her symptoms alone. If individual motivation plays an important part at the outset, it will nevertheless be seriously influenced by the relationship between the doctor and his/her patient. It is one of the rare factors for which a positive correlation with compliance can be observed, as shown by practically all medical psychological research. These well-known classical medical notions meet with average values of non-compliance, which may vary widely. These data are proof of the wide gap existing between what is expected and what is actually achieved. This discrepancy is felt by the patient as well as by his/her therapist, and involves many other factors linked to culture, to the medical and religious background, to medical anthropology, and to the real or supposed proprieties of the Pharmakon. Our study aims at synthesizing the most recent data concerning this issue, which is still at the core of numerous debates and hardly ever tackled in French language literature. Based on the summary of these data and on the testimony of 135 persons involved in this dynamic (patients, families, doctors, nurses, members of pharmaceutical industry), we shall try to obtain a better understanding of what happens between the patient and his/her therapists from an anthropological viewpoint. Social isolation remains one of the greatest losses in mental disease. For many authors, people with schizophrenia can have no friends, no spouse, and sometimes no family. Two thirds of the patients with schizophrenia return to their parents' house when discharged from a hospital after the first psychosis. Family members generally receive very little education as to what they can expect. They
\end{abstract}

\footnotetext{
* Auteur correspondant.

Adresse e-mail : palazzolo@free.fr (J. Palazzolo).
} 
may not even know the importance of medication compliance. Family members are the primary victims of violence from psychotic individuals, usually their own son or daughter, and most families cannot believe their own son or daughter would be capable of such violence. Although families are usually the main care givers at the beginning of schizophrenia they often find their experience very frustrating for a number of reasons, and consequently relationships suffer. Family education and support have been shown to improve outcomes considerably and family education is the second strongest factor in relapse prevention. Without education and good relapse prevention families often disintegrate. Most of the homeless mentally ill in downtown city cores have lost their family relationships. It is not a reflection on their families so much as the lack of adequate treatment and support. The families tried again and again but finally lost their sick relative. These reflections show how information of and about the psychiatric patient is necessary, and underlines the importance of the relationships between the patient and his family. Our article also insists on this theme which is rarely developed in the literature.

(C) 2007 Elsevier Masson SAS. Tous droits réservés.

Mots clés : Anthropologie de la santé ; Gestion autonome de la médication ; Observance ; Psychiatrie ; Relation médecin-malade

Keywords: Compliance; Empowerment; Medical Anthropology; Psychiatry; Therapeutic relationship

\section{Introduction}

En étudiant les troubles mentaux en fonction des groupes ethniques ou culturels, ainsi que la place qu'ils occupent dans l'équilibre social, l'approche anthropologique de la pathologie psychiatrique et de la thérapeutique qui s'y rapporte nous apprend que chaque collectivité possède ses propres modèles de déviance, et que l'on est toujours fou par rapport à une société donnée [1,9,18,32]. Cette dynamique est peut-être aussi ancienne que la médecine elle-même. Et dans un tel contexte, il nous est apparu important de nous intéresser à la manière dont les traitements psychotropes sont respectés et suivis à la suite de la prescription. De nombreux patients réclament d'ailleurs le « droit à une gestion autonome de leur médication » [16,23].

La gestion autonome de la médication demeure un concept qui, à ce jour, ne fait pas l'objet d'une définition et d'une connaissance partagées par l'ensemble des acteurs intervenant en santé mentale et en anthropologie. Un certain nombre d'auteurs ont tenté de mieux cerner cette problématique, mais il n'existe à l'heure actuelle aucun consensus la concernant $[4,6,7,40,43,44]$.

La définition la plus structurée - c'est-à-dire offrant l'espace à un débat potentiellement constructif tout en se référant à une réalité à laquelle sont confrontés les usagers est la suivante : «Une gestion autonome signifie le droit et la possibilité pour toute personne recevant des médicaments d'être informée sur les raisons, la pertinence, les effets secondaires et le mode d'utilisation de la médication prescrite ; d'être avertie des alternatives possibles à cette médication et d'être encouragée à les utiliser si nécessaire; de pouvoir négocier avec le prescripteur le type de médicament, la dose, la fréquence de prise [...] de décider de diminuer - voire d'arrêter — toute médication totalement ou partiellement, définitivement ou provisoirement, en étant accompagnée dans ce processus par le médecin prescripteur ou adressée à un autre professionnel de la santé capable et désireux de faire cet accompagnement » [34].

Il est important de rappeler que la gestion autonome de la médication n'est pas une fin en soi, mais un soutien de l'individu centré sur un processus thérapeutique qui permet de traiter la souffrance de manière novatrice. Toutefois, un tel cheminement demande la participation de l'ensemble des intervenants et nécessite une collaboration étroite avec les familles, les proches, voire les associations d'usagers. Cette problématique prend une dimension fondamentale dans le débat anthropologique actuel concernant l'évolution du réseau en santé mentale, son objectif prioritaire de réhabilitation psychosociale et la prise en considération des conditions nécessaires à sa mise en place [2,12,39]. Il nous est donc apparu important d'aborder ce concept selon des angles différents. Après avoir mis en exergue la situation particulière de la psychiatrie vis-à-vis de la gestion autonome de la médication, nous envisagerons certaines solutions, tout en insistant sur les enjeux anthropologique et sociologique d'une telle approche.

Divers témoignages nous permettent de constater que chaque groupe d'intérêt possède une vision qui lui est propre concernant cette problématique. Notre approche a pour principal objectif de faire part des connaissances et de l'expérience des différents acteurs concernés par une gestion autonome de la médication.

Il apparaît donc intéressant de faire une analyse systémique du problème, en collectant le point de vue des acteurs et en recherchant leur logique, les points d'achoppement, les points aveugles et divergents, de sorte qu'il sera alors possible d'élaborer des conclusions qui éclairent le système et les stratégies d'acteurs au sein de ce système [8,14,17]. Dans cette optique, nous avons donc fait une enquête de terrain, tout d'abord comme participant, comme acteur qui prend une distance de chercheur pour entendre les avis, les positions, et comprendre la dynamique à l'œuvre dans la problématique.

Le présent article se veut une synthèse de divers témoignages concernant la prescription d'une médication psychotrope. Les acteurs impliqués nous ont chacun livré leur point de vue, l'ensemble des propos recueillis permettant de dresser un véritable état des lieux de la situation actuelle en psychiatrie concernant la problématique de la gestion autonome du traitement. Ont été interrogés des patients, des familles et des proches, des soignants, ainsi que des membres de l'industrie pharmaceutique.

\section{Méthodologie de l'enquête}

L'étude s'est déroulée du $1^{\mathrm{er}}$ septembre au 31 décembre 2004 au sein du Centre Hospitalier Sainte-Marie de Nice et des 
structures extérieures (centres de consultation) qui en dépendent. Cent trente-cinq personnes ont été interrogées selon des entretiens non directifs. Cette population se répartit comme suit : 35 patients suivis en Centre Médico-Psychologique, 35 sujets proches de ces patients (un membre de la famille de chaque personne interrogée), 29 infirmiers travaillant aussi bien dans l'intra- que dans l'extrahospitalier, 24 psychiatres hospitaliers (14) et libéraux (dix), 12 membres de l'industrie pharmaceutique (Fig. 1).

Les sujets inclus dans l'étude ont été sélectionnés en fonction de leur capacité à aborder la problématique de la prescription d'une médication psychotrope. Les entretiens ont été enregistrés, puis regroupés par thématique, ce qui a permis d'en faire la synthèse.

\section{Résultats}

\subsection{Le point de vue des patients}

La majorité des sujets interrogés (83\%) soulignent que la prescription de médicaments est quasi systématique au sein des unités d'hospitalisation en psychiatrie, et que par ailleurs le manque d'information concernant les psychotropes demeure un problème majeur. Une insuffisance de renseignements sur les buts thérapeutiques recherchés, les effets secondaires éventuels, les risques d'interaction des principes actifs entre eux ou avec d'autres substances, est ainsi déplorée par les malades. De plus, les patients se questionnent sur l'obtention du consentement de l'usager concernant son traitement : ce consentement est-il vraiment libre et éclairé ? Dans le même ordre d'idées, selon les commentaires de nombreux sujets interrogés $(86 \%)$, le malade peut difficilement discuter ou négocier le type, voire le dosage de la médication prescrite. Il se voit donc parfois astreint à la même posologie de principe actif alors que la crise est terminée et qu'il a pris congé de l'hôpital. Des lacunes sont également signalées au niveau de la qualité du suivi médical inhérent au traitement médicamenteux (une réévaluation régulière de la nécessité de poursuivre la thérapeutique n'étant alors pas systématique selon les dires des patients).

De nombreux usagers $(80 \%)$ mettent en exergue la problématique d'une certaine surmédication, des dosages trop élevés entraînant divers effets secondaires handicapants qui rendent l'individu amorphe — le terme de « zombie » revient

\section{Population interrogée}

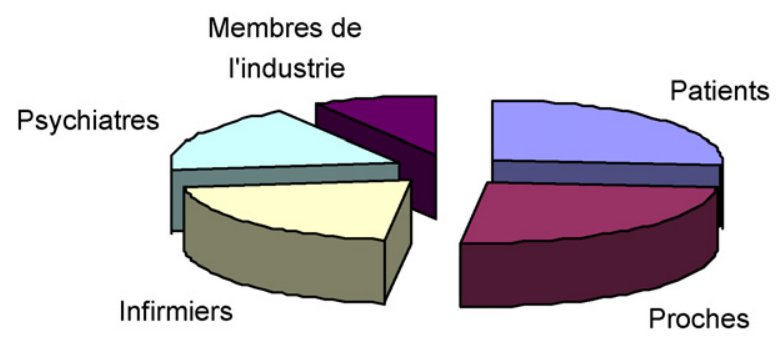

Fig. 1. Population soumise à l'enquête. souvent dans le discours —, entraînent sa marginalisation, sa chronicisation ; il s'ensuivrait la construction d'une véritable identité de malade mental. Et dans une telle optique, les usagers se sentent invalidés, ayant peu de pouvoir sur leur propre trajectoire psychosociale.

Nos interlocuteurs mentionnent également le fait que le sujet n'a pas toujours l'opportunité ou la détermination de faire valoir sa volonté d'obtenir un traitement comportant un minimum, voire une absence de psychotropes. Par ailleurs, le sentiment que les prescripteurs sont peu sensibles aux demandes de sevrage médicamenteux est un thème récurrent. Les besoins exprimés à ce niveau proviendraient surtout des usagers sous neuroleptiques, neuroleptiques dont les effets secondaires sont souvent décrits comme pénibles (alors qu'actuellement c'est plutôt la consommation de benzodiazépines qui préoccupe les médecins et les pharmaciens). Devant un refus du psychiatre traitant concernant le fait de répondre à une demande de sevrage médicamenteux, certains usagers $(8 \%)$ ont selon leurs dires dû procéder à la diminution ou à l'arrêt de leur médication sans aide appropriée et sans connaissances pertinentes, étant alors victimes de symptômes inhérents à une « rupture thérapeutique sauvage ». Certains autres patients $(11 \%)$ ont pu bénéficier d'un suivi effectué par un médecin généraliste, voire par un psychiatre plus conciliant à leurs yeux. Enfin, quelques sujets $(6 \%)$ font part d'un soutien apporté par leur pharmacien, voire par diverses associations d'usagers lorsqu'ils ont voulu arrêter ou réduire la posologie de leurs médicaments. Pour ce qui concerne les rares demandes effectuées auprès de centres spécialisés en addictologie, une telle démarche apparaît tout à fait inappropriée, les structures et les modalités de prise en charge n'étant pas adaptées à cette problématique.

Quoi qu'il en soit, la demande de sevrage existe, et que ce dernier soit réalisé avec ou sans suivi médical strict, un arrêt ou une diminution du traitement psychotrope doivent s'effectuer dans le cadre d'une évaluation psychopathologique régulière et en respectant certaines règles. Certains sujets interrogés (11\%) décrivent plusieurs cas de rupture totale de soins qui se sont soldés par une réhospitalisation rapide en milieu spécialisé.

En définitive, de nombreux patients (31\%) soulignent le manque cruel d'alternatives à la médication pure, soit parce que ces alternatives ne sont pas suggérées, pas encouragées par les soignants, voire inconnues des médecins, soit parce qu'elles ne sont pas valorisées; en contrepartie, le mythe de l'existence d'une pilule miracle est encore bien présent au sein de l'opinion publique. Les malades interrogés mentionnent également le manque de ressources pouvant être utilisées par la communauté en tant qu'alternatives ou compléments d'un traitement pharmacologique. L'ensemble des témoignages recueillis apparaît toutefois unanime concernant la nécessité de privilégier une approche psychothérapique, dont l'objectif tend vers une réhabilitation psychosociale. Si le traitement pharmacologique est indispensable, il doit alors être minimal, n'entravant en aucune manière les possibilités de réinsertion socioprofessionnelle de l'individu. 


\subsection{Le point de vue des familles et des proches}

Les familles sont souvent très impliquées dans la prise en charge et le soutien d'un parent atteint de maladie mentale. Malgré cela, ces dernières reçoivent selon leurs dires peu ou pas de renseignements concernant la pharmacothérapie en cours. Pour la majorité des familles interrogées (71\%), il existe d'importantes difficultés de communication avec le psychiatre traitant. En conséquence, elles se tournent de plus en plus fréquemment vers les pharmaciens, qui généralement, répondent favorablement à leur besoin d'information concernant les médicaments prescrits.

Les familles et les proches sont des témoins privilégiés de l'évolution clinique de l'usager, évolution en lien avec la médication suivie. Parallèlement au fait de composer avec les manifestations de la maladie mentale, les familles et les proches subissent les réactions symptomatiques, les malaises éventuels du patient aux prises avec les effets secondaires de son traitement psychotrope. Si le sujet décide de cesser brusquement toute médication sans suivi médical approprié, les proches craignent une rechute et interviennent dans de nombreux cas auprès du malade et du médecin traitant pour que la prise en charge pharmacologique et psychothérapique se poursuive.

Nos interlocuteurs signalent par ailleurs que les familles sont parfois aux prises avec un dilemme opposant deux idéologies distinctes : d'un côté les tenants de la psychiatrie biologique préconisent une approche psychopharmacologique, et de l'autre certains soignants se revendiquant hors de cette mouvance prônent un abord plus alternatif, relevant exclusivement du champ psychosocial. Cependant, il apparaît que les contrastes s'estompent dans de nombreux cas de figure, une vision plus intégrative se développant au sein de l'environnement psychiatrique actuel.

Mais dans de tels cas de figure, les familles sont alors livrées à elles-mêmes, et il est par ailleurs souligné qu'il existe une insuffisance manifeste pour ce qui concerne le soutien et l'accompagnement des proches, insuffisance se retrouvant dans le peu de structures mises à disposition des patients (hébergement, centre d'accueil de crise, structures d'aide à l'insertion socioprofessionnelle...). En conséquence, les familles doivent pallier ces manques par un engagement sans faille auprès de leur parent malade. Dans cette optique, le risque d'épuisement est majeur.

\subsection{Le point de vue des infirmiers}

Selon $69 \%$ de nos interlocuteurs, une approche thérapeutique centrée uniquement sur l'aspect biologique des troubles n'est pas satisfaisante car une telle démarche, entreprise isolément, ne tient pas compte des dimensions psychologiques, sociales ou spirituelles de l'individu. Par ailleurs, une mauvaise articulation des diverses composantes du soin est évoquée, tout comme un manque de concertation et de collaboration entre les professionnels de la santé ; une certaine incohérence et une fragmentation du réseau de soin sont également mises en exergue.
Un manque de disponibilité et d'accessibilité des médecins psychiatres est rapporté. Il est reproché à ces derniers de recourir systématiquement à la chimiothérapie, chimiothérapie alors considérée comme une solution privilégiée face à la souffrance psychique. Mais il semble que l'influence du mythe de la pilule miracle, voire la pression des usagers ou des proches pour obtenir une prescription médicamenteuse, soient parfois des éléments prépondérants à prendre en compte dans ce contexte. De plus, la recherche continue et inévitable d'une certaine performance - médicale, économique, sociale dans la prise en charge des patients, telle l'évolution permanente de la politique de santé mentale, la réduction constante du nombre de lits et la diminution de la durée moyenne de séjour à l'hôpital, favoriserait — en partie — cette pratique.

Selon $83 \%$ des soignants interrogés, les demandes de diminution de traitement ne sont généralement pas prises en compte — bien souvent à juste titre — si elles sont directement adressées au psychiatre au cours de l'hospitalisation. Ces demandes sont davantage prises en considération - à distance de la crise - par l'omnipraticien ou le psychiatre libéral, qui sont alors perçus comme étant plus ouverts et plus disponibles pour collaborer à une telle démarche. Les pharmaciens sont également des professionnels dont le rôle est qualifié de majeur par les infirmiers que nous avons consultés : donnant volontiers des renseignements et des conseils aux usagers et aux familles, certains pharmaciens collaborent par ailleurs avec des médecins pour faciliter l'arrêt ou la diminution de psychotropes chez les patients qui en ont exprimé le besoin.

Pour $86 \%$ des soignants interrogés, il est important de souligner que les psychiatres sont de plus en plus conscients de la problématique de surmédication, principalement pour ce qui concerne la prescription d'anxiolytiques et d'hypnotiques. C'est ainsi qu'une évolution est notée dans les services hospitaliers, les ordonnances des patients comportant de moins en moins de benzodiazépines.

Quoi qu'il en soit, dans la majorité des témoignages recueillis $(79 \%)$, le sevrage médicamenteux est considéré comme une entreprise difficile et complexe à mettre en œuvre ; de nombreuses lacunes théoriques existent, plus particulièrement au niveau des connaissances concernant les antidépresseurs et les antipsychotiques — peut-on alors réellement dans ce cas parler de sevrage ? Les expériences de sevrage aux benzodiazépines sont quant à elles plus nombreuses, et l'expertise en ce domaine est plus étendue.

Plusieurs sujets interviewés (52\%) soulignent que les infirmiers craignent souvent de donner des informations au patient concernant les médicaments en cours ou les éventuelles possibilités de sevrage, en avançant le fait qu'ils n'ont pas suffisamment de connaissances en psychopharmacologie et en clinique. Cependant, il leur arrive de déplorer certaines prescriptions, sans toutefois en référer systématiquement au médecin responsable (exemples : benzodiazépine poursuivie au long cours, neuroleptique sédatif dispensé à posologie élevée, associations médicamenteuses cavalières...).

Il est par ailleurs déploré que certains praticiens, ayant accepté la demande de sevrage ou de réduction de posologie, 
l'effectuent d'une manière inappropriée en ne respectant pas les recommandations retrouvées dans la littérature.

Enfin, les infirmiers s'interrogent sur la tendance à prescrire davantage de médicaments aux enfants, aux personnes âgées, aux femmes enceintes; l'accessibilité aux nouveaux médicaments (avec moins d'effets secondaires désagréables) est également rapportée.

\subsection{Le point de vue des psychiatres}

Les praticiens interrogés rappellent que l'expression clinique des symptômes allégués par le patient, constatée par le médecin et rapportée à une étiologie, conduit au pouvoir thérapeutique - celui du médicament en l'occurrence - qui est au centre de la relation médecin-malade. L'ordonnance acquiert ses lettres de noblesse dans une valeur symbolique représentée par les caractéristiques du médicament, son nom, sa couleur, ses modalités d'administration, son prix... et l'espoir de guérison qui y est associé. Pour $79 \%$ des psychiatres interviewés, la gestion autonome de la médication est une réalité culturelle, sociale, et actuelle dans notre pays. Elle flirte malheureusement parfois avec l'automédication — rappelonsle, $85 \%$ des Français auraient recours à un traitement pharmacologique sans avis médicalisé selon une étude Sofres menée sur un échantillon représentatif de 1135 personnes : ainsi est réalisée une « automédication », avec une proportion de $17 \%$ de molécules «non-prescrites » (500 millions d'unités) sur l'ensemble des médicaments vendus en 1998 (2,9 milliards d'unités vendues en officine).

L'utilisation ou l'arrêt spontanés d'un psychotrope par tout individu sous sa propre responsabilité n'est pas forcément critiquable... même si cette pratique est en contradiction avec les règles modernes de bon usage du médicament, de traçabilité, de qualité des soins... Pour $75 \%$ des psychiatres interrogés, une gestion autonome de la médication non étayée n'est pas sans comporter des risques pour la santé du patient, risques liés par exemple à la nature même du produit, à l'inadaptation éventuelle de son utilisation, à la compétition ou à la synergie avec un autre médicament, à une posologie inadaptée, au manque de connaissances basiques de l'individu qui y a recours — soit pour lui-même, soit, ce qui peut être plus grave, pour autrui... Ainsi, certains psychotropes induisant des troubles de la vigilance peuvent initier des accidents lors du travail ou de la conduite sur route, voire majorer un péril lié à une consommation d'alcool associée. .

La majorité des témoignages recueillis (88\%) soulignent que si une gestion autonome de la médication s'avère utile dans l'attente d'un avis médical — pour pallier temporairement un effet secondaire important, elle risque en revanche de devenir dangereuse si elle échappe trop longtemps à une consultation psychiatrique ou si elle est utilisée de manière irréfléchie alors que l'état psychique n'est pas encore stabilisé. La prudence nécessite donc une éducation de l'usager, notamment par une information pertinente. Les acteurs de santé ont ici une place importante mais non exclusive, et lors d'une prescription le praticien ne doit pas laisser ignorer le danger du psychotrope si celui-ci était utilisé hors du cadre pour lequel il est recommandé.

\subsection{Le point de vue des membres de l'industrie pharmaceutique}

La très grande influence des compagnies pharmaceutiques est régulièrement dénoncée par les médias. Il est ainsi déploré par certains auteurs le fait que la recherche dans le domaine des substances médicamenteuses soit sous le contrôle de ces entreprises, dont l'objectif principal est la vente de produits pharmacologiques. Par ailleurs, il est reproché à l'information dispensée sur les médicaments de ne pas être nécessairement objective ou complète, puisqu'elle provient de l'industrie pharmaceutique. Cela étant dit, les représentants de ces compagnies sont généralement plus disponibles et souvent mieux formés que les psychiatres cliniciens pour informer l'usager et le médecin sur un plan purement pharmacologique. Selon $83 \%$ des membres de l'industrie pharmaceutique que nous avons interrogés, il est évident que l'utilisation de la publicité doit être contrôlée, car il n'existe a priori pas de réelle dynamique critique rigoureuse dans les médias pour ce qui concerne la promotion des médicaments, ce qui n'aide pas à l'ébranlement du mythe de la pilule miracle au sein de la population. Il est par ailleurs déploré que certains médicaments dits « de confort », pouvant être obtenus sans ordonnance, puissent être proposés directement au consommateur qui, bien souvent, n'a pas les connaissances nécessaires pour évaluer le discours publicitaire et son contenu.

\section{Discussion : les problèmes soulevés et les solutions proposées par nos interlocuteurs}

Parmi les sujets que nous avons interrogés, nous avons pu constater une certaine homogénéité de perception en ce qui concerne les problèmes liés à l'usage de la médication psychotrope, ainsi qu'au niveau des solutions alternatives proposées. La majorité de nos interlocuteurs s'entendent sur un objectif commun qui est d'atteindre un meilleur équilibre dans l'investissement des dimensions biopsychosociales telles que décrites par Mauss [30].

Nous avons également observé que l'utilisation du terme « sevrage » soulève certaines inquiétudes liées aux risques de rechute, de réhospitalisation... En revanche, la notion de gestion autonome de la médication, telle qu'élaborée en introduction de cet article, rallie l'ensemble des répondants. Ce dernier terme apparaît en effet plus nuancé : il englobe les notions d'optimisation de la thérapeutique, de dose minimale efficace, de gestion des effets secondaires — voire d'arrêt — du psychotrope, d'accompagnement et de traitement individualisés.

Ainsi, ces rencontres nous ont permis de cerner les problèmes inhérents à la prise régulière d'un traitement psychotrope, et d'envisager les solutions proposées par nos interlocuteurs. Les données recueillies ont été regroupées en trois rubriques : l'usager, les médicaments, le prescripteur. 


\subsection{L'usager}

\subsubsection{Qui sont les usagers de médicaments psychotropes?}

Les usagers de psychotropes appartiennent selon nos interlocuteurs à toutes les tranches d'âge et à toutes les classes sociales. Des stimulants sont prescrits aux enfants, aux adultes ; les femmes apparaissent comme les plus grandes consommatrices d'antidépresseurs et d'anxiolytiques; on parle même de « génération Prozac ». Les personnes âgées consomment non seulement des médicaments à visée somatique, mais également de nombreux psychotropes. Les sujets souffrant d'une pathologie mentale invalidante et chronique prennent beaucoup de médicaments, entre autres divers antipsychotiques. Ce phénomène de consommation élargie des psychotropes apparaît inquiétant pour la majorité des individus interrogés.

\subsubsection{Primauté de la personne et empowerment}

En Amérique du Nord, la politique de santé mentale met de plus en plus l'accent sur la notion de primauté de la personne, et le principe d'empowerment social (la réappropriation du pouvoir par l'individu et par la communauté) est mis en avant, cette dynamique concernant autant les usagers en santé mentale que l'ensemble des professionnels impliqués dans le réseau de soins et le réseau social.

Lorsqu'il est question de la médication, plusieurs problèmes se posent et plusieurs défis s'imposent. L'un de ces problèmes est le respect du consentement libre et éclairé du sujet. Le consentement libre et éclairé fait référence à la transmission d'une information accessible, c'est-à-dire suffisamment vulgarisée, neutre, portant sur la pertinence, la durée, les effets secondaires, la posologie du traitement, ainsi que sur l'impact social éventuel (travail, entourage. . .). Le consentement libre et éclairé suppose des alternatives éventuelles et/ou un complément à la médication. Le fait de ne proposer au patient qu'une solution pharmacologique pour traiter ses problèmes de santé mentale est extrêmement réducteur. Notons aussi que de nombreux sujets (67\% des personnes interrogées) reprochent aux prescripteurs d'instaurer un traitement à une certaine posologie sans livrer au malade la moindre explication.

Cette notion de consentement libre et éclairé va beaucoup plus loin que le simple fait de faire un étalage des possibilités thérapeutiques envisagées. Elle invite le praticien à s'engager dans une démarche de collaboration et de coopération qui amène le patient à être informé de la médication qui lui est prescrite ; par ailleurs, le médecin doit s'assurer que le sujet a bien assimilé cette information et qu'il est en mesure de prendre la décision qu'il jugera la plus adaptée. À la fin de ce processus, la personne concernée peut décider de suivre ou non la prescription effectuée, tout en ayant la possibilité de continuer la prise en charge.

Nos divers entretiens nous ont permis de mettre en exergue le fait que pour l'ensemble des sujets interrogés l'empowerment est un objectif difficile à atteindre lorsque l'individu concerné consomme de fortes doses de médicaments pouvant altérer sa conscience. Et lorsque celui-ci décide de réduire ou d'arrêter son traitement, il ne trouve pas toujours facilement une écoute attentive de la part d'un soignant prêt à l'accompagner dans une telle démarche. De nombreux sujets $(56 \%$ des personnes interrogées) nous ont fait part de leurs difficultés concernant ce point : l'arrêt de la médication s'est effectué dans plusieurs cas de figure de manière anarchique, sur une courte période, avec parfois apparition d'importants symptômes de sevrage.

\subsubsection{L'insertion sociale}

La plupart de nos interlocuteurs (82\% des sujets interrogés) soulignent que les personnes qui souffrent de maladie mentale et qui consomment des psychotropes à forte dose sont confrontées à certaines difficultés en termes de réinsertion. L'une de ces difficultés est la rareté des ressources communautaires qui offrent des services spécifiques d'insertion sociale (travail, logement. . .). Un autre désagrément évoqué est la survenue d'un certain état léthargique induit par une éventuelle posologie excessive de médicament sédatif. Ce ralentissement psychomoteur est un frein important à l'initiative, à l'insertion sociale et à l'empowerment. Les effets secondaires pénibles à supporter pour le sujet ont été mentionnés comme étant l'une des raisons de l'arrêt intempestif des médicaments.

\subsubsection{Le support nécessaire à la personne qui vise une gestion autonome de sa médication}

L'ensemble des personnes interrogées insistent sur le fait que le premier pas vers une gestion autonome de la médication est avant tout de reconnaitre que celle-ci est non seulement un choix, mais également un droit fondamental de l'individu. À partir du moment où cette reconnaissance est effective, un profond changement de la pratique clinique habituelle s'opère. Par ailleurs, au vu de l'évolution actuelle de la législation, on peut constater que de plus en plus de patients revendiquent ce droit à la gestion autonome de leur médication.

Quatre-vingt-quatre pour cent de nos interlocuteurs mettent en exergue le fait suivant : lorsqu'un malade désire réduire la posologie ou changer de médicament, il se heurte régulièrement au refus du praticien de l'aider et de l'accompagner dans sa demande. Il s'avère donc indispensable d'obtenir l'appui et le soutien d'un autre psychiatre ou du médecin traitant dans une telle démarche. Actuellement, nombreuses sont les personnes qui arrêtent simplement leur médication, faute de support pour un changement de traitement.

La gestion autonome de la médication n'est pas une fin en soi, mais plutôt un moyen d'accompagner le patient vers une certaine indépendance. Il est essentiel que ce dernier ait accès à des services spécialisés au sein de la communauté pour l'épauler dans son choix. Le suivi de secteur est perçu par l'usager et sa famille comme l'une des meilleures options au niveau de l'accompagnement. Les groupes d'entraide sont également considérés comme des ressources utiles à la personne qui désire entreprendre une démarche d'autonomisation. À l'intérieur de ces groupes, certains participants ont vécu de difficiles expériences de rupture de soins. En conséquence, ces derniers pourront apporter un soutien efficace et significatif.

Lorsqu'il est question de gestion autonome de la médication, la lacune la plus importante - selon la plupart des sujets interrogés $(77 \%)$ - a trait à l'information : il semble que le 
praticien ne fournisse que rarement des renseignements adaptés concernant les médicaments qu'il prescrit et, lorsqu'il le fait, ceux-ci sont généralement insuffisants. D'autre part, l'information fournie n'est jamais neutre. Par conséquent, le consentement libre et éclairé ne pourrait pas s'exercer dans de telles conditions.

Soixante-treize pour cent de nos interlocuteurs souhaitent vivement une plus large circulation de l'information. Celle-ci doit être transmise au patient, à sa famille, à son entourage, afin que tous puissent s'unir autour de l'individu et le supporter dans sa démarche de gestion autonome de sa médication. Plus l'information sera répandue, plus le réseau relationnel et affectif se mobilisera et apportera le soutien nécessaire.

Pour la majorité des sujets interrogés (89\%), une plus grande concertation entre les différents acteurs (l'usager, ses proches, les ressources communautaires, le prescripteur, le pharmacien...) est essentielle, et doit être associée à une importante disponibilité de ces derniers.

\subsection{Les médicaments}

Nos interlocuteurs insistent sur les points suivants :

- il existe en France une certaine tendance à la surmédication ; cette dernière est régulièrement soulignée par les médias, et les conséquences sur les usagers sont préjudiciables ;

- il est possible de recenser un type de médicament pour chaque catégorie d'usagers, et il semble qu'il y ait eu ces dernières années un accroissement et un rajeunissement de la population qui consomme des psychotropes ;

- les données scientifiques sont consensuelles pour ce qui concerne la durée et les modalités de diminution d'un traitement anxiolytique, alors qu'en ce qui concerne les neuroleptiques les opinions restent partagées.

Une grande partie des patients qui demandent à bénéficier d'une gestion autonome de leur médication sont sous neuroleptique ou antipsychotique. L'un des problèmes soulevés par les sujets interrogés à ce propos est le manque d'information sur ce type de psychotropes: les effets secondaires, la posologie, la pertinence de la prescription, la manière de prendre son traitement, la durée... sont autant de questions parfois restées sans réponse. Si bien que certains usagers psychotiques en viennent à se demander si leur apragmatisme est secondaire au traitement ou à l'évolution naturelle de la maladie. Enfin, il est également mis en exergue le fait que l'information qui concerne les interactions médicamenteuses (médicament-médicament, médicament-alcool, médicament-drogues...) est extrêmement difficile à obtenir de la part des médecins.

Toutes les personnes rencontrées s'accordent à souligner l'importance d'évaluer régulièrement et de limiter la survenue des effets secondaires, dont certains peuvent être handicapants pour le malade et sources d'arrêt prématuré de la médication.

Une minorité de sujets interrogés (19\%) se dit désenchantée, le traitement prescrit ayant suscité des attentes non réalistes. Certains évoquent même le fantasme de la «pilule miracle », les résultats obtenus s'avérant alors décevants. Dans ce contexte, le rôle omniprésent de l'industrie pharmaceutique, tant dans le secteur de la recherche que dans celui de la pratique médicale, est lourdement décrié. Certains usagers (38\%) pensent alors que les résultats des études sont biaisés, ces derniers n'étant cliniquement pas à la hauteur de leurs attentes. Plusieurs (59\%) évoquent un conflit d'intérêt dans lequel se placeraient les compagnies pharmaceutiques : « Elles produisent, font la promotion et vendent des médicaments ; alors comment accorder du crédit aux recherches qu'elles réalisent ou à l'information qu'elles font circuler? Ces recherches devraient être effectuées uniquement par des scientifiques neutres tels les universitaires, et ne devraient pas concerner essentiellement la psychopharmacologie. Elles devraient porter aussi sur les alternatives non médicamenteuses ou les formes d'aide complémentaires. »

\subsection{Le prescripteur}

Les médecins prescripteurs sont montrés du doigt lorsqu'on parle de biologisation croissante des problèmes de santé mentale et de surmédication. Cependant, plusieurs de nos interlocuteurs praticiens $(63 \%)$ sont confrontés à un double discours de la part de certains patients, qui revendiquent leur statut d'individu malade tout en évoquant un dérèglement essentiellement biologique. Les dimensions psychosociales risquent alors d'être sous-investies par le soigné et, consécutivement, par le soignant. Dans un tel contexte, il est souvent reproché au psychiatre son automatisme à prescrire et son manque de disponibilité pour écouter : «Chaque problème a sa pilule ! » Le praticien doit donc, dans ce type de situation, rester vigilant et favoriser l'établissement d'une relation fondée sur l'empathie.

De plus, bon nombre d'usagers $(78 \%)$ reprochent aux médecins d'être à l'origine d'une prescription excessive de psychotropes. Les patients s'interrogent sur le maintien fréquent de la même posologie lors de la période de crise et lors de la période de stabilisation. Enfin, ils constatent que les prescripteurs travaillent dans certains cas en vase clos : est à nouveau soulignée l'importance d'une étroite collaboration avec le patient, ses proches, les associations d'usagers, les intervenants sociaux, les pharmaciens, etc. Une meilleure information du malade et de son entourage apparaît comme une nécessité pour l'ensemble des sujets interrogés, cette information ayant une place majeure dans l'optimisation du suivi psychiatrique instauré au long cours.

De nombreux usagers (64\%) mettent en exergue un manque de soutien de la part du psychiatre dans le cadre d'une éventuelle gestion autonome de la médication. La personne se heurte dans bien des cas au refus du praticien de l'aider et de l'accompagner dans cette voie; une diminution anarchique rapide de la posologie de certains psychotropes est alors parfois mal vécue, divers sujets interrogés relatant une expérience extrêmement pénible, avec apparition d'importants symptômes de sevrage (le terme de « sevrage sauvage » est employé à de nombreuses reprises dans ce contexte). Enfin, une communication et un soutien de meilleure qualité semblent être notés avec le « médecin de famille ». 
Soixante-quinze pour cent de nos interlocuteurs soulignent qu'en ce qui concerne la gestion autonome des médicaments, il s'avère essentiel de mieux informer les prescripteurs, soit par le biais d'une formation continue, soit par celui de séances de supervision plus performantes associées à un questionnement approfondi concernant les pratiques psychiatriques actuelles.

\section{En pratique clinique, quelles stratégies mettre en ouvre ?}

Le maintien d'une stabilisation de l'état clinique d'un sujet atteint d'une pathologie psychique nécessite la mise en œuvre d'une stratégie thérapeutique aux multiples facettes, dont l'observance est l'un des principaux éléments [35,37]. Malheureusement, un patient peut présenter une recrudescence de sa symptomatologie psychotique malgré le respect d'une bonne observance médicamenteuse [27]. La méta-analyse effectuée par Gilbert et al. en 1995 [20], recensant 66 études, retrouve un taux de décompensation de $53 \%$ parmi les sujets qui ont arrêté leur traitement, mais il s'avère que ce taux est encore de $16 \%$ parmi les malades ayant pris correctement leur médication, et ce sur une période de 9,7 mois. Dans le même ordre d'idée, l'enquête réalisée par Weiden et Olfson [46] met en évidence que dans la première année suivant leur sortie, les patients réadmis à l'hôpital pour cause d'échec thérapeutique sont deux fois plus nombreux que ceux qui sont réadmis pour cause d'observance aléatoire, cette proportion ayant tendance à s'inverser au cours de la deuxième année suivant la sortie. Il est important de souligner qu'il est parfois difficile de faire la part entre un échec thérapeutique et un défaut d'observance médicamenteuse, la conséquence étant alors une augmentation de la posologie de l'antipsychotique prescrit [28]. À l'inverse, l'explication simpliste consistant à évoquer d'emblée un défaut présumé d'observance dans le cadre de réadmissions répétées d'un malade peut être à l'origine de certains préjugés de la part des soignants, et ainsi générer un contre-transfert négatif [36,47].

Le maintien d'une vigilance toute particulière concernant l'inscription du sujet dans le projet de soin : la plupart des études réalisées sur ce thème mettent en évidence le fait que la survenue d'événements de vie stressants, tels que la perte d'un emploi, une rupture sentimentale ou le décès d'un proche, peuvent être à l'origine d'une nouvelle décompensation en dehors de tout défaut d'observance médicamenteuse [46]. Certains profils d'évolution clinique, caractérisés par une rémission partielle de la symptomatologie délirante, une importante intensité des éléments déficitaires, de l'isolement psychosocial et de l'apragmatisme, des effets secondaires extrapyramidaux handicapants, sont également plus à risque de présenter un épisode de décompensation, et ce malgré la prise correcte du traitement antipsychotique [29].

Ainsi, il est important de souligner que la recrudescence de la symptomatologie délirante, parfois consécutive à la présence d'une observance médicamenteuse aléatoire, peut également être en lien avec des difficultés relationnelles particulièrement dans les cas où les émotions ne sont pas exprimées, et/ou lorsque les capacités de verbalisation sont médiocres $[5,26,38]-$, des rendez-vous de consultation psychothérapique manqués de manière récurrente [45], voire un abus de substances [49]. Le maintien d'une vigilance toute particulière concernant l'inscription du sujet dans le projet de soin permet donc au clinicien de prévenir la survenue d'une éventuelle nouvelle décompensation psychotique, puisqu'en moyenne il s'écoule entre trois et six mois entre l'arrêt de la prise en charge psychiatrique et les premiers prodromes signant la réactivation psychotique [51]. Young et al. [50] soulignent l'importance du rôle du pharmacien dans le maintien de cette alliance thérapeutique nécessaire.

L'apparition de prodromes: la mise en évidence de prodromes signant une éventuelle réactivation de la symptomatologie psychotique est un élément essentiel de la prise en charge; ces indicateurs ont été étudiés par de nombreux auteurs, et on peut ainsi souligner que la survenue d'un brusque changement d'humeur, ou d'un retrait affectif, voire de troubles du comportement chez un sujet schizophrène, peuvent laisser présager une nouvelle décompensation [51]. Thomas [45] a cependant noté que $50 \%$ des épisodes de décompensation n'étaient pas précédés de signaux d'alerte, et qu'à l'inverse dans $53 \%$ des cas l'apparition de symptômes qualifiés de « prodromes » ne donnait pas lieu à une nouvelle rechute lorsqu'ils n'étaient pas traités. Malgré cela, il est recommandé de réinstaurer un traitement psychotique ou de majorer la posologie de la médication en cours lorsque des prodromes apparaissent [35], une telle mesure permettant dans la plupart des cas d'obtenir une amélioration rapide de l'état clinique du patient [53].

Généralement, ces prodromes appartiennent au registre psychotique, mais une importante atypicité peut également se retrouver [31]. Dans un tel contexte, Zrinyi [54] préconise d'entraîner le malade à reconnaître lui-même les prémices d'une éventuelle décompensation. Il est évident que les modalités d'expression de tels signaux d'alerte sont spécifiques à chaque patient, et qu'elles doivent être intégrées dans la trajectoire clinique des sujets, le recours systématique à une attitude prédéterminée et codifiée devant être évité [52]. Diverses recommandations sont proposées par certains auteurs [34], mais tous s'accordent à dire que la prise en compte de l'appréciation des proches du malade est un point essentiel dans le cadre de la mise en évidence de prodromes pouvant signer une nouvelle décompensation psychotique.

La prévision d'une éventuelle décompensation : bien que la plupart des épisodes de décompensation surviennent de manière assez brutale et imprévisible, certains auteurs ont pu mettre en exergue le fait que la fréquence de survenue de tels cas de figure pouvait être diminuée de façon notable grâce au maintien d'une bonne alliance thérapeutique, elle-même favorisée par une relation médecin-malade de qualité. Ainsi, Young et al. [50] soulignent que $79 \%$ des épisodes de décompensation survenus dans le cadre de leur étude ont pu être pressentis grâce à la réalisation d'entretiens réguliers avec les patients et leur famille, ces épisodes ayant rapidement été endigués grâce à la majoration de la médication antipsychotique. Subotnik et Nuechterlein [in 35] ont pour leur part anticipé de deux à quatre semaines, et ce dans $70 \%$ des cas, la 
survenue d'une recrudescence de la symptomatologie psychotique des sujets participant à leur étude.

Certains facteurs prédictifs biologiques ont pu être mis en évidence, mais leur utilisation en pratique clinique n'est pas encore d'actualité [34].

La prise en compte des facteurs environnementaux : la qualité de vie des patients schizophrènes a un impact indéniable sur l'observance médicamenteuse [4], et sa préservation permet de diminuer notablement le risque de décompensation psychotique [49]. L'information donnée aux sujets concernant leur maladie et leur traitement est bien souvent limitée [37], et si la mise en œuvre de modules éducationnels apporte des résultats encourageants dans le cadre de la réhabilitation psychosociale, du respect de l'alliance thérapeutique et de la qualité de vie des malades, d'importantes avancées restent à réaliser dans ce domaine [6]. De Prospero et Riffle [in 34] ont montré que l'utilisation des thérapies cognitivocomportementales permettait de réduire la durée d'hospitalisation des patients schizophrènes; ces résultats confirment les conclusions de Lecompte et Pelc [in 34], ainsi que celles de Guimon [in 34]. Par ailleurs, des travaux similaires ont été effectués avec des sujets schizophrènes toxicomanes, les perspectives envisagées étant là encore tout à fait encourageantes [12]. Il semble donc que l'éducation des malades et de leurs proches concernant, entre autres, la gestion du traitement et l'appréhension des symptômes psychotiques, apporte un bénéfice certain dans le cadre du respect de l'observance médicamenteuse et la stabilisation de l'état clinique [49]. L'implication de la famille du sujet dans la prise en charge au long cours est un élément important, concourant à l'obtention et au maintien d'une alliance thérapeutique de qualité [36] ; ainsi, Penn et Mueser [in 34] proposent au malade et à ses parents un programme éducationnel structuré au sein duquel l'observance médicamenteuse est favorisée par le biais d'un renforcement positif continu.

\section{Conclusion}

Selon nos interlocuteurs, il s'avère impérieux :

- de reconnaître et d'encourager une gestion autonome de la médication chez les usagers ;

- de considérer la gestion autonome de la médication comme un droit fondamental du patient ;

- de fournir au malade, à son entourage et à la population en général une information adaptée et compréhensible sur les psychotropes ;

- de s'interroger de manière approfondie sur les pratiques psychiatriques actuelles;

- de reconnaître et recenser les diverses expériences de gestion autonome de la médication, et de regrouper l'information afin de développer une certaine expertise dans le cadre de l'obtention d'une dose minimale efficace. Les sujets ayant bénéficié d'un sevrage médicamenteux pourraient alors faire part de leurs propres observations et les partager dans le cadre de projets pilotes associés au développement d'alternatives thérapeutiques ;
- de consolider un véritable partenariat autour de la problématique de la gestion autonome de la médication et du développement d'alternatives thérapeutiques (en y associant le patient, l'entourage, les groupes d'entraide, les associations d'usagers, les médecins généralistes, les psychiatres, les pharmaciens...).

Ainsi, au vu des différents témoignages recueillis, il apparaît nécessaire d'insister sur une définition préalable de la culture en tant que système de signification $[3,24,25,33,48]$. La maladie et les théories explicatives qui tentent d'en rendre compte sont bien évidemment présentes, mais elles ne résument pas l'ensemble de la démarche suivie ici. En fait, c'est la pathologie en tant qu'expérience culturelle et subjective (illness) qui est au cœur de l'enquête, et non la catégorie médicale ou traditionnelle (disease) $[19,42]$. L'opposition de ces deux conceptions permet de rendre compte de la perception culturelle mais également pragmatique de la souffrance psychique telle qu'elle est perçue par les malades, leurs proches, et les soignants [13,15,21,22]. Cette perception culturelle de la maladie correspond à l'évidence aux catégories autochtones, tandis que l'approche pragmatique laquelle domine la plupart des stratégies thérapeutiques proposées aux patients, quelle que soit la culture de ces derniers - fait intervenir l'ensemble des facteurs relationnels et environnementaux qui constituent le sens commun et conditionnent la vie quotidienne $[10,11,41]$. À ce titre, l'approche anthropologique du concept de gestion autonome de la médication permet de mieux cerner les frontières de celui-ci, ou en tout cas d'en préciser la définition.

\section{Références}

[1] Augé M. L'anthropologie de la maladie. L'Hommece:hsp sp025/: Revue Française d'Anthropologie 1986;26:81-90.

[2] Benoist J. Soigner au pluriel : essais sur le pluralisme médical. Paris: Karthala; 1996.

[3] Bourdieu P, Wacquant LJD. Pour une anthropologie réflexive. Paris: Seuil; 1992.

[4] Burner M. Le médecin et le médicament. "La compliance". Psychol Med 1990;22:502-4.

[5] Cailloux-Cohen S, Vigneault L. Aller-retour au pays de la folie. Montréal: Les Éditions de L'Homme; 1997.

[6] Cohen D. Les « nouveaux » médicaments de l'esprit, marche avant vers le passé? Sociol Soc 1996;28:17-33

[7] Cohen D. Quand les bonnes intentions se substituent à l'analyse critique. Sociol Soc 1996;28:39-43.

[8] Crozier M, Friedberg E. L'acteur et le système. Les contraintes de l'action collective. Paris: Seuil; 1996.

[9] Devereux G. Essais d'ethnopsychiatrie générale. Paris: Gallimard; 1977.

[10] Devereux G. Ethnopsychanalyse complémentariste. Paris: Flammarion; 1972.

[11] Fabrega H. Medical anthropology. Biennal Review of Anthropology 1971;11(6):167-229.

[12] Fainzang S. Médicaments et société. Le patient, le médecin et l'ordonnance. Paris: PUF; 2001.

[13] Fassin D. Idéologie, pouvoir et maladie. In: Les maux de l'autre. Paris: L'Harmattan; 1996.

[14] Foster G, Anderson B. Medical anthropology. New York: Wiley; 1978.

[15] Foucault M. Naissance de la clinique. Paris: PUF; 1963.

[16] Freidson E. La profession médicale. New York: Dodd and Mead; 1970.

[17] Good B. Comment faire de l'anthropologie médicale ? Médecine, rationalité et vécu.. Paris: Les Empêcheurs de Penser en Rond; 1998. 
[18] Herzlich C. Médecine moderne et quête de sens : la maladie signifiant social. In: Auge M, Herzlich C, editors. Le sens du mal. Anthropologie, histoire, sociologie de la maladie. Paris: Éditions des Archives Contemporaines; 1983. p. 189-211.

[19] Kleinman A. Writing at the margin: Discourse between anthropology and medicine. Berkeley: University of California Press; 1995.

[20] Gilbert PL, Harris MJ, Mac Adams LA. Neuroleptic withdrawal in schizophrenic patients: A review of the literature. Arch Gen Psychiatry 1995;52:173-88.

[21] Kleinman A. Patients and healers in the context of culture. An exploration of the borderland between anthropology medicine and psychiatry. Berkeley: University of California Press; 1980.

[22] Laplantine F. Anthropologie médicale. Paris: Payot; 1986.

[23] Le Breton D. Anthropologie du corps et modernité. Paris: PUF; 2000.

[24] Levi-Strauss C. Le regard éloigné. Paris: Plon; 1983.

[25] Levi-Strauss C. Anthropologie structurale. Paris: Plon; 1973.

[26] Luhrmann TM. Of two minds. Knopf; 2000.

[27] Mann JJ. How médication compliance affects outcome. Psychiatr Ann 1986;16:567-70.

[28] Marchais P. Le nouvel esprit psychiatrique. Métamorphose et développement de la psychiatrie clinique. Paris: Frison-Roche; 1996.

[29] Martino P. La fiction thérapeutique. Psychosomatique 1993;32:65-71.

[30] Mauss M. Sociologie et anthropologie. Paris: PUF; 2004.

[31] Murphy JM. Anthropology and psychiatric epidemiology. Acta Psychiatr Scand 1994;14(3):48-57.

[32] Nathan T, Stengers I. Médecins et sorciers. Paris: Les Empêcheurs de Penser en Rond; 1995.

[33] Nathan T. La folie des autres. Traité d'ethnopsychiatrie clinique. Paris: Dunod; 1986.

[34] Palazzolo J. Communication et observance dans la relation thérapeutique en psychiatrie. Thèse d'Anthropologie, Nice, 2004a.

[35] Palazzolo J. Observance médicamenteuse et psychiatrie. Paris: Elsevier; 2004.

[36] Palazzolo J. Dire pour vivre - Pathologies psychiques : témoignages au quotidien. Paris: Ellébore, Collection Champs Ouverts; 2004.

[37] Palazzolo J. Au-delà des maux - Paroles oubliées ou l'importance de l'écoute. Paris: Ellébore, Collection Champs Ouverts; 2003.
[38] Rechtman R. Anthropologie médicale et psychiatrie : de nouveaux paradigmes de recherche. Pour la Recherche 1998;17:2-9.

[39] Sargent C, Johnson T. Medical anthropology: Contemporary theory and method. Westport: Praeger; 1996.

[40] Scheper-Hugues N. The primacy of ethical: Propositions for a militant anthropology. Curr Anthropol 1995;36:409-20.

[41] Scheper-Hugues N. Three propositions for a critically applied medical anthropology. Soc Sci Med 1990;30:189-97.

[42] Scheper-Hugues N, Lock MM. The mindful body: A prolegomenon to future work in medical anthropology. Med Anthropol Q 1987;1:6-41.

[43] Scotch NA. Medical Anthropology. In: Siegel H, editor. Biennal Review of Anthropology. Standford: Standford University Press; 1963.

[44] Tessier L, Clément M. La réadaptation psychosociale en psychiatrie : défis des années 1990. Montréal: Gaëtan Morin; 1992.

[45] Thomas M. Establishing a legal compliance program. QRC Advis 1997;13:4-8.

[46] Weiden PJ, Olfson M. Cost of relapse in schizophrenia. Schizophr Bull 1995;21:419-29.

[47] Wright EC. Non-compliance or how many aunts has Matilda? Lancet 1993;342:909-13.

[48] Young A. Misapplying medical anthropology in multicultural settings. Santé Culture Health 1990;7:197-208.

[49] Young JL, Spitz RT, Hillbrand M, Daneri G. Medication adherence failure in schizophrenia: a forensic review of rates, reasons, treatments, and prospects. J Am Acad Psychiatry Law 1999;27:426-44.

[50] Young JL, Zonana HV, Shepler L. Medication noncompliance in schizophrenia: Codification and update. Bull Am Acad Psychiatry Law 1986;14:105-22.

[51] Yung AR, Mac Gorry PD. The prodromal phase of first-episode psychosis: Past and current conceptualizations. Schizophr Bull 1996;22:353-70.

[52] Zagury D. Clinique, éthique et mutations sociales. Inf Psychiatr 1998;74:47-56.

[53] Zito JM, Routt WW, Mitchell JE, Roerig JL. Clinical characteristics of hospitalized psychotic patients who refuse antipsychotic drug therapy. Am J Psychiatry 1985;142:822-6.

[54] Zrinyi M. The influence of staff-patient interactions on adherence behaviours. EDTNA ERCA J 2001;26:13-6. 